\title{
Behavioral Drive versus Behavioral Inertia in Evolution: A Null Model Approach
}

\author{
Raymond B. Huey, ${ }^{1, \star}$ Paul E. Hertz, ${ }^{2, \dagger}$ and B. Sinervo ${ }^{3, \ddagger}$
}

1. Department of Biology, Box 351800, University of Washington, Seattle, Washington 98195;

2. Department of Biology, Barnard College, Columbia University, New York, New York 10027;

3. Department of Ecology and Evolution, University of California, Santa Cruz, California 95064

Submitted August 8, 2001; Accepted August 27, 2002;

Electronically published February 11, 2003

ABstract: Some biologists embrace the classical view that changes in behavior inevitably initiate or drive evolutionary changes in other traits, yet others note that behavior sometimes inhibits evolutionary changes. Here we develop a null model that quantifies the impact of regulatory behaviors (specifically, thermoregulatory behaviors) on body temperature and on performance of ectotherms. We apply the model to data on a lizard (Anolis cristatellus) and show that thermoregulatory behaviors likely inhibit selection for evolutionary shifts in thermal physiology with altitude. Because behavioral adjustments are commonly used by ectotherms to regulate physiological performance, regulatory behaviors should generally constrain rather than drive evolution, a phenomenon we call the "Bogert effect." We briefly review a few other examples that contradict the classical view of behavior as the inevitable driving force in evolution. Overall, our analysis and brief review challenge the classical view that behavior is invariably the driving force in evolution, and instead our work supports the alternative view that behavior has diverse-and sometimes conflicting-effects on the directions and rates at which other traits evolve.

Keywords: Anolis cristatellus, behavior, clines, null model, stasis, thermoregulation.

\footnotetext{
* E-mail: hueyrb@u.washington.edu.

† E-mail: phertz@barnard.edu.

₹ E-mail: sinervo@biology.ucsc.edu.
}

Am. Nat. 2003. Vol. 161, pp. 357-366. (c) 2003 by The University of Chicago. 0003-0147/2003/16103-010281\$15.00. All rights reserved.
A shift into a new niche or adaptive zone is, almost without exception, initiated by a change in behavior. The other adaptations to the new niche, particularly the structural ones, are acquired secondarily. With habitat and food selection-behavioral phenomena-playing a major role in the shift into new adaptive zones, the importance of behavior in initiating new evolutionary events is self-evident. (E. Mayr [1963, p. 604])

By behavior organisms can effectively damp out heterogeneity in the physical environment. ... Indeed this dampening of external environmental heterogeneity seems to be one of the major trends of evolution. (R. N. Brandon [1988, p. 65])

Evolutionary biologists classically view behavior as an important pacemaker or driver of evolutionary change. Indeed, many argue that "behavioral drive" usually initiates evolutionary shifts in morphology, physiology, or ecology (Schmalhausen 1949; Mayr 1959, 1963; Hardy 1965; Wyles et al. 1983; Plotkin 1988; Wcislo 1989) or that behavioral shifts often promote speciation (Lande 1981; West-Eberhard 1989; Boake 1994). Nevertheless, some other biologists-predominantly physiological and functional ecologists - note that behavior can sometimes inhibit rather than drive evolutionary change in other traits (Elton 1927; Bogert 1949; Bartholomew 1964; Hertz 1981; Hertz and Huey 1981; Coyne et al. 1983; Wake et al. 1983; Brandon 1988; Odling-Smee 1988; Aboitiz 1990; van Damme et al. 1990). These authors note that regulatory behaviors (e.g., behavioral thermoregulation) often dampen the impact of environmental variation on organisms, thereby minimizing the intensity of selection on other traits.

Our purpose here is not to challenge the validity of the classical view (e.g., Mayr 1963) that behavior is an important driver of evolutionary change. Instead, our primary goal is to develop and apply a quantitative null model of thermoregulation and show that the thermoregulatory behavior of a lizard likely inhibits selection for an evolutionary shift in thermal physiology with altitude. For completeness, we briefly review a few other issues and studies that reiterate a view that behavior has complex and 
sometimes even competing roles in evolution (Hardy 1965; Bateson 1988). Behavior may frequently drive evolution, but it sometimes maintains the status quo. Moreover, behavior can maintain the status quo for one trait while simultaneously driving the evolution of others (Bogert 1959).

\section{The Classical View: Behavior as a Driver of Evolution}

The belief that behavior usually drives evolutionary change has always been a central premise of evolutionary biology (Lamarck 1809; Darwin 1859). In a more recent era, this view was championed by Mayr (1959, 1963, 1974) and subsequently supported by others (see critical reviews in Bateson 1988; Plotkin 1988; Wcislo 1989; West-Eberhard 1989; Gittleman et al. 1996). The basic argument derives from the assumption that behavioral shifts are relatively labile (see Gittleman et al. 1996; Wimberger and de Queiroz 1996) and can expose an organism to novel experiences or environments and thus to novel selection pressures, which in turn trigger a cascade of evolutionary changes in morphology, physiology, or ecology (Bateson 1988; Wcislo 1989; West-Eberhard 1989). A familiar example involves sexual selection, in which changes in mate-choice behaviors drive the rapid evolution and diversification of bizarre male ornaments (Lande 1981; Kirkpatrick 1982; Andersson 1994).

The logic of this argument is appealing and widely accepted. Nevertheless, Plotkin (1988) challenged the empirical base supporting the universality of behavioral drive. He commented (p. 144), "The notion that behavior is an evolutionary pacemaker has been around for at least four decades. Yet only one set of empirical studies by A. C. Wilson and his colleagues has been published, and that very recently. Without further empirical support, the claim that behavior is an evolutionary pacemaker will not survive another 40 years." Wcilso (1989) and West-Eberhard (1989) disagreed and reviewed diverse examples that support the classical view. Nevertheless, these apparent examples need to be reanalyzed within a phylogenetic framework (Wcislo 1989; Harvey and Pagel 1991; Gittleman et al. 1996; Wimberger and de Queiroz 1996).

Several others have also challenged the view that behavior is always a driving force in evolution (Bogert 1949; Bartholomew 1964; Hertz 1981; Hertz and Huey 1981; Coyne et al. 1983; Wake et al. 1983; Brandon 1988, 1990; Endler and McLellan 1988; Odling-Smee 1988; Ryan 1990; van Damme et al. 1990). These workers have argued that behavior can inhibit rather than drive evolution. Several note that organisms are not necessarily at the mercy of environmental change or gradients. Indeed, many use regulatory behaviors (e.g., habitat selection or thermoregulation) to buffer or even to modify the selective environ- ment that they actually experience. Such behavioral adjustments can reduce-perhaps even eliminate- the environmental variation that would be experienced by a nonregulating organism, thereby reducing selection pressures for evolutionary change. We refer to this phenomenon as the "Bogert effect" in honor of the late Charles M. Bogert, who helped pioneer the study of behavioral thermoregulation in ectotherms (Cowles and Bogert 1944) and who (Bogert 1949, 1959) enunciated this view long ago.

\section{Thermoregulatory Behavior as an Inertial Factor in Evolution}

To show how behavior can be an inertial factor, we describe a simple hypothetical example. We then use this example as a springboard to develop a null model that quantifies the extent to which thermoregulatory behavior may inhibit selection for evolutionary change in thermal physiology along an altitudinal gradient. Note that we assume that the shifts in thermoregulatory behavior with altitude are completely facultative.

Consider a hypothetical example of selection on the thermal physiology of a lizard distributed along an altitudinal gradient. Individuals living at low altitude will be exposed to warmer environmental temperatures on average than will individuals living at higher altitude (Porter 1989). However, whether body temperatures also decline with altitude depends on the effectiveness of the lizard's thermoregulatory behavior. One extreme on a continuum of thermoregulatory capacities would be a nonregulating lizard ("null" lizard) that selects habitats, perches, and times of activity randomly with respect to environmental factors that influence body temperature (Heath 1964; Huey et al. 1977; Grant and Dunham 1990; Hertz 1992b; Hertz et al. 1993; Willis and Beaupre 2000). Null lizards living at low altitude would thus necessarily have higher body temperatures on average than would null lizards living at high altitude. Because body temperature has a profound influence on performance and fitness (see insert in fig. 1; Huey and Stevenson 1979; Bennett 1980; Christian and Tracy 1981; Huey 1982; Huey and Berrigan 2001), selection should favor the evolution of progressively lower "optimal" performance temperatures with increasing altitude (Hertz et al. 1983; Huey and Bennett 1987; Bennett et al. 1990; Huey and Kingsolver 1993). However, marked gene flow among populations might reduce a response to selection (e.g., Sultan and Spencer 2002).

In contrast, consider a thermoregulating lizard that actively adjusts its behavior to the local thermal environment. Very likely, it will occupy shaded habitats and perches at low altitude but more open habitats and perches at high altitude (Rand 1964; Adolph 1990a), and it will shift its times of activity with altitude as well (Hertz 1981; 


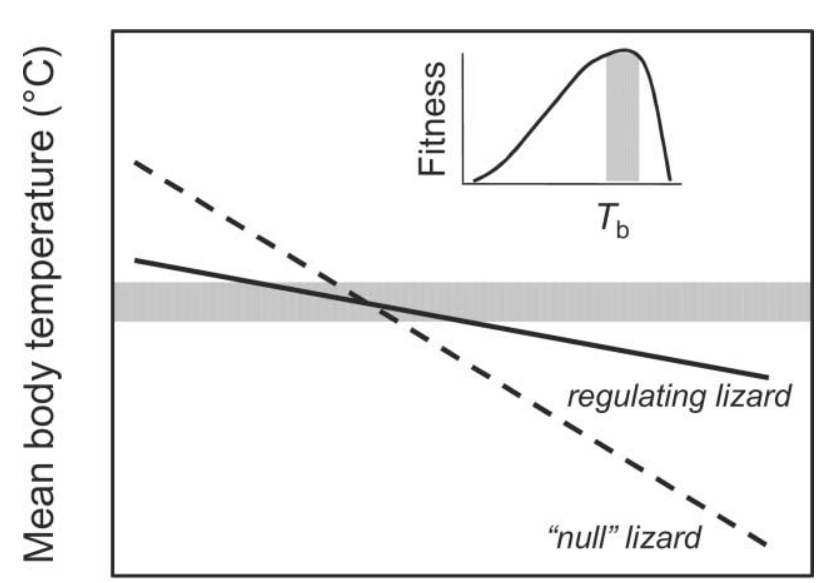

Altitude

Figure 1: Hypothetical impact of thermoregulation on altitudinal changes in body temperature $\left(T_{\mathrm{b}}\right)$ of a lizard. The effect of $T_{\mathrm{b}}$ on fitness is shown in an insert, and the temperature range that maximizes fitness is shown in gray both in the insert and in the main graph; $T_{\mathrm{b}}$ will decline less steeply with altitude (and deviate less from optimal temperature zone) for a thermoregulating lizard than for a nonregulating (null) lizard.

Hertz and Huey 1981; Grant and Dunham 1990). As a consequence, its body temperatures will decline less precipitously with altitude than would be the case for a nonregulating lizard (Huey and Webster 1976; Hertz 1981; Grant and Dunham 1990; van Damme et al. 1990; Hertz et al. 1993). Selection may still favor a lowered optimal temperature for high-altitude populations of thermoregulators (because lizards are not perfect thermoregulators), but decline in optimal temperature with altitude will be much reduced, relative to that for a null lizard. Thus, thermoregulatory behaviors will buffer the potential impact of selection induced by geographic (or seasonal) variation in the thermal environment.

To our knowledge, Bogert $(1949,1959)$ was the first to propose explicitly that behavioral shifts could inhibit evolutionary change. He reached this conclusion after studying geographic variation in the body temperatures of lizards (Sceloporus, Cnemidophorus) living in diverse climates. To his apparent surprise, Bogert found that body temperatures of these lizards varied little geographically and were largely independent of local environmental temperatures. Indeed, body temperatures of Sceloporus living in hot lowland deserts were quite similar to those of species living in cloud forests. (Note: Brattstrom [1965] reanalyzed Bogert's data and argued that mean body temperatures did, in fact, vary with altitude, but in a little-known paper, Bogert [1980] strongly rebutted Brattstrom's analysis and conclusions.) Bogert (1949, 1959) believed that the observed lack of geographic variation in body temperature must reflect the effectiveness of behavioral thermoregulation, which would enable lizards to maintain similar body temperatures in diverse thermal environments. $\mathrm{He}$ also recognized that the resultant lack of geographic variation in body temperatures would in turn blunt selection for geographic variation in the thermal sensitivity of physiology; thus, he clearly appreciated that behavior can constrain the evolution of other traits.

\section{A Null Model of the Impact of Behavioral Thermoregulation}

Bogert's conclusions were perceptive. Here, we formalize and extend his insights (Bogert 1949, 1959) into a null model by applying conceptual and methodological approaches developed in subsequent years. In principle, our model is general and can be modified to analyze the buffering impact of many other kinds of behaviors. We apply our model to data derived from extensive studies of the lizard Anolis cristatellus along an altitudinal gradient in Puerto Rico. This species shows conspicuous altitudinal shifts in behaviors that directly influence body temperatures (e.g., choice of habitats, microhabitats, times of activity, basking frequency; see Rand 1964; Schoener and Schoener 1971; Huey and Webster 1976; Hertz 1992a, $1992 b$ ). Because these lizards make similar adjustments to daily and seasonal variation in their thermal environment (Hertz 1992a, 1992b), observed behavioral differences with altitude are probably facultative.

To evaluate quantitatively whether thermoregulatory behaviors reduce selection for altitudinal shifts in thermal physiology, one must develop a null model that contrasts the predicted patterns of selection on thermoregulating versus nonthermoregulating (null) lizards (Heath 1964; Huey et al. 1977; Hertz et al. 1993; Willis and Beaupre 2000). Such a model requires answers to several critical questions.

First, how much would body temperatures of hypothetical, nonthermoregulating (null) lizards change with altitude (fig. 1)? Although nonthermoregulating mutants exist in Drosophila (Sayeed and Benzer 1996) and Caenorhabditis (Hedgecock and Russell 1975), no such mutants exist for lizards. Nevertheless, we can predict the body temperatures of null lizards by measuring operative temperatures $\left(T_{\mathrm{e}}\right)$, which closely approximate equilibrium body temperatures of ectotherms at particular perch sites (Bakken 1992). Such operative temperatures integrate the net impact of the radiative and convective environments on organismal heat balance; they can be estimated either with biophysical calculations (Roughgarden et al. 1981; Porter and Tracy 1983) or by measuring the temperatures inside physical models (often of copper) that mimic the size, shape, posture, and color of the species under study 
(Bakken 1992). Of particular relevance here is the fact that the distribution of $T_{\mathrm{e}}$ at randomly selected perch sites at a given locality represents the null distribution of predicted body temperatures of nonthermoregulating lizards (Grant and Dunham 1990; Hertz 1992b; Hertz et al. 1993; Willis and Beaupre 2000). Thus, by monitoring changes in mean $T_{\mathrm{e}}$ with altitude, we can readily estimate the impact of altitude on the body temperatures of nonthermoregulating lizards.

Second, how much do body temperatures $\left(T_{\mathrm{b}}\right)$ of real lizards change with altitude (fig. 1)? This question is easily answered by sampling $T_{\mathrm{b}}$ of lizards at multiple altitudes. If behavioral thermoregulation effectively reduces geographic variation in body temperature (Hertz et al. 1993), then the altitudinal differences in body temperatures observed for real lizards will be significantly smaller than those predicted for null lizards (fig. 1).

Third, does thermoregulation reduce the impact of altitudinal change on predicted physiological performance (Huey 1983), not just on $T_{b}$ ? If so, then the altitudinal variation in performance will be substantially less for thermoregulating lizards than for null lizards (Hertz et al. 1993); thus, selection for altitudinal variation in thermal sensitivity will likely be blunted.

To answer these questions, we reanalyze data from our prior work with A. cristatellus in Puerto Rico (Huey and Webster 1976; Huey 1983; Hertz 1992a, 1992b; Hertz et al. 1993). Note that for simplicity we focus only on average temperatures and average performance, even though differences in the distributions of body temperatures can affect patterns of selection (D. Bauwens, personal communication). We report operative temperatures of $A$. cristatellus at low and at high altitude (Hertz 1992a, 1992b); these data were measured at randomly selected perch sites during the lizards' usual times of activity in both January and August, the coolest and warmest months, respectively. Body temperatures of field-active lizards were collected simultaneously at the same sites and seasons (Hertz 1992a, $1992 b$ ). Altitudinal patterns of $T_{\mathrm{b}}$ documented here are consistent with those previously reported for this species (e.g., Rand 1964; Huey and Webster 1976).

To determine whether observed changes in body temperature would have an impact on physiological performance, we analyzed data on the thermal sensitivity of sprint speed in A. cristatellus (Huey 1983). These data serve as a convenient proxy for the overall sensitivity of physiological performance to temperature (Huey and Stevenson 1979; Bennett 1980; Huey 1983; van Berkum 1986; van Damme et al. 1990; Hertz et al. 1993). We used curvefitting algorithms (van Berkum 1986) to predict the relative performance (i.e., percentage of maximal speed achieved) of a real or a null lizard with a specified body temperature (Huey 1983; Hertz et al. 1993). Maximal speed occurs at $29.5^{\circ} \mathrm{C}$ (Huey 1983).

Our data suggest that nonthermoregulating lizards would experience more than a threefold greater variation in body temperature with altitude and season than do real lizards. Mean $T_{\mathrm{e}}$ differed by $13.8^{\circ} \mathrm{C}$ between the $5-\mathrm{m}$ site in August and the 1,150-m site in January (fig. $2 A$ ), but mean $T_{\mathrm{b}}$ of real lizards sampled simultaneously at the same sites and seasons differed by only $7.3^{\circ} \mathrm{C}$ (fig. $2 A$ ).

Null and thermoregulating lizards also differ in the degree to which altitude and season influence predicted sprint performance (fig. $2 B$ ). At the lowland site in summer, thermoregulating lizards should sprint well $(84.6 \%$ of maximal speed), but null lizards would be hyperthermic and would sprint poorly (only $66.0 \%$ of their potential). At the high-elevation site in January, thermoregulating lizards would still be warm enough to run quickly $(84.4 \%$ of maximal speed), but null lizards would be both cold and slow (68.9\% of maximal speed).

Body temperatures and sprint performances of real lizards are thus much less affected by altitudinal and seasonal variations in environmental temperatures than are those of hypothetical nonthermoregulating lizards (fig. 2). If selection operates on sprint performance (Christian and Tracy 1981; Bennett and Huey 1990; Jayne and Bennett 1990) or on traits with similar thermal sensitivities, then selection for altitudinal variation in the thermal sensitivity should be much less intense for real lizards than for nonthermoregulating lizards.

We attribute the reduced altitudinal and seasonal variation in body temperature and in performance of real lizards (relative to null lizards; fig. 2) to the effectiveness of thermoregulation by the real lizards. At high elevation, these lizards shift to more open habitats and microhabitats, which are relatively warm (Rand 1964; Schoener and Schoener 1971; Huey and Webster 1976; Hertz 1992a, $1992 b$ ); in addition, they are often more active at midday and during sunny weather, avoiding activity at the coolest times (Hertz 1992b). Morphological shifts (e.g., in color and/or size) with altitude might also mitigate environmental pressures (cf. Bogert 1949, 1959), but their impact on body temperatures is almost certainly minimal, relative to the impact of behavior (Porter and Tracy 1983; Stevenson 1985).

We assume that the altitudinal shifts in thermoregulatory behavior by $A$. cristatellus are facultative, not fixed. This is highly likely: individual lizards conspicuously shift their behavior (time of activity, microhabitat, and habitat selection) in response to daily and seasonal changes in the thermal environment (Heath 1965; Huey et al. 1977; Grant and Dunham 1990; Hertz 1992b). Moreover, plasticity of thermoregulatory behavior is almost certainly ancestral for lizards (Cowles and Bogert 1944; Avery 1982; Huey 1982), 


\section{Anolis cristatellus in Puerto Rico}
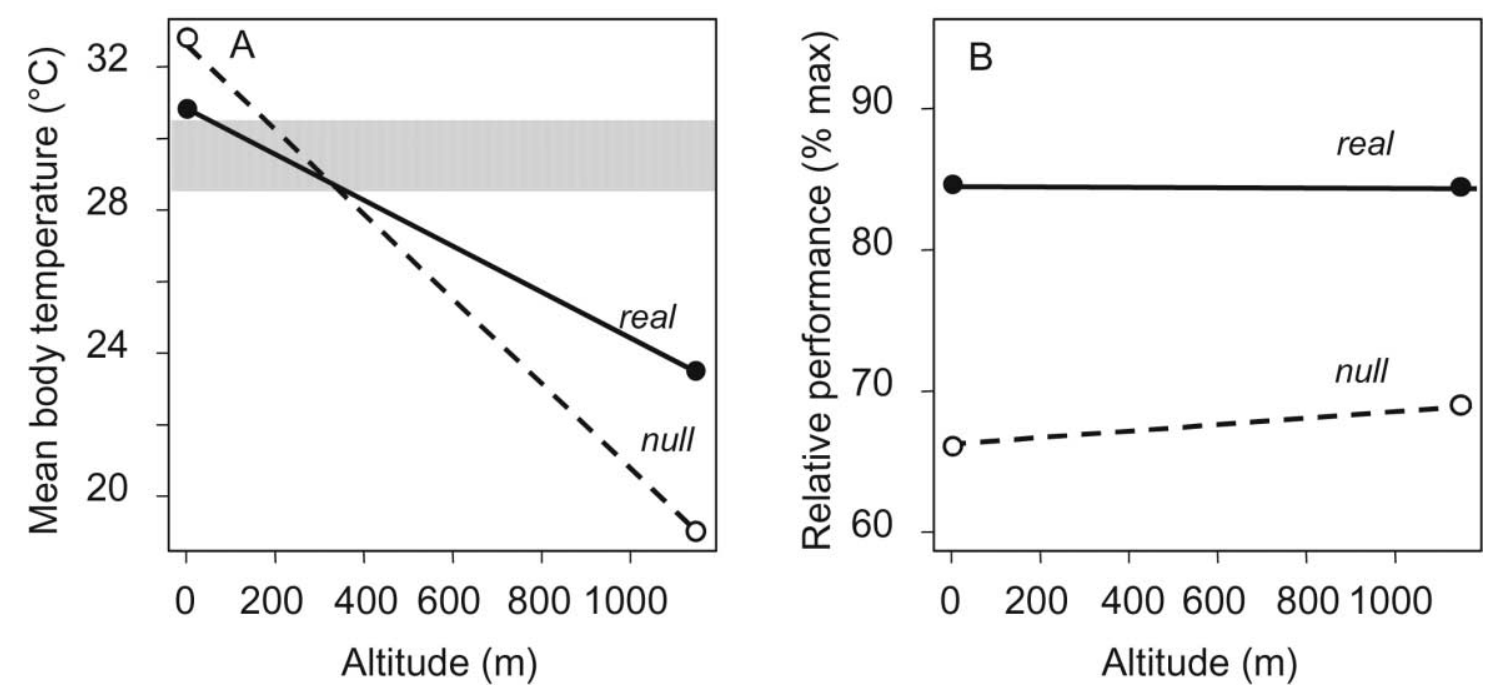

Figure 2: A, Mean body temperature ( $T_{\mathrm{b}}$ ) of real lizards (Anolis cristatellus; solid circles) and of null lizards (open circles) at two altitudes in Puerto Rico. The preferred temperature zone is shown in gray. Mean $T_{\mathrm{b}}$ declines less steeply (and is closer to the thermal preference zone) for real than for null lizards. B, Predicted average relative sprint performance (percentage of maximum) for real and null lizards at two altitudes. Performance is much lower for null lizards, which are too hot at low altitude and too cold at high altitude. Data from (or calculated from) Huey (1982), Hertz (1992a, 1992b), and Hertz et al. (1993).

since behavioral thermoregulation is a characteristic of most lizard taxa (Avery 1982) and is no doubt maintained because such behavioral shifts enhance performance and likely fitness in thermally fluctuating environments (Christian and Tracy 1981; Hertz et al 1993). Nevertheless, reciprocal transplants (Kiester et al. 1975) or commongarden studies with naive lizards would be necessary to test this assumption. An appropriate model would be Adolph's $(1990 a, 1990 b)$ studies of habitat selection in Sceloporus occidentalis and Sceloporus graciosus, two lizards that are distributed along an elevation gradient in the San Gabriel Mountains of California. Lizards at low altitude typically select much higher perches than do lizards at high altitude, undoubtedly because operative temperatures near the ground at low altitude are stressfully high (Adolph 1990a). In a common-garden laboratory arena, naive hatchlings of both species from low and high altitude selected similar perch heights (Adolph 1990b), confirming that observed shifts in perch height with altitude (Adolph 1990a) are plastic. Moreover, gene flow among populations is high (S. Adolph, personal communication), supporting the assumption that observed shifts in behavior with altitude are facultative.

Our analysis provides quantitative support for Bogert's view (1949) that thermoregulatory behavior can buffer selection on thermal physiology. Similar examples are known for other lizards (e.g., Grant and Dunham 1990; van Damme et al. 1990) or for other ectotherms. For example, Jones et al. (1987) showed indirectly that the temperatures apparently experienced by developing Drosophila melanogaster changed very little with altitude, possibly because of altitudinal shifts in the oviposition sites selected by females.

\section{Haldane's Dilemma}

J. B. S. Haldane was one of the first evolutionary biologists in the last century to think quantitatively about rates of evolution. He was puzzled to observe that rates of evolution observed in fossil assemblages were substantially lower than those found in artificial selection experiments (Haldane 1949). Levinton (1988) described this observation as "Haldane's dilemma" and proposed that the pattern might merely be an artifact of different sampling intervals in different kinds of studies. (Fossils are typically sampled over relatively long time periods; hence, the derived rates of evolution will be averages that underestimate maximal rates.) Gingerich (1983) offered a similar explanation.

Behavioral inertia might provide an additional but nonexclusive explanation of Haldane's dilemma. If lineages shift behaviors in response to temporal changes in environmental conditions, then the actual selection pressures for physiological or morphological change might be much weaker than expected if based solely on the magnitude of 
the environmental change. Hence, the response to selection will also be less than expected, slowing rates of evolution under natural conditions (Wake et al. 1983; Stearns and Hoekstra 2000).

\section{Related Issues}

We would be remiss not to mention a few other roles of behavior, especially as they pertain to behavior as a driving force in evolution. Here we briefly review a few examples showing the complexity of roles for behavior in evolution.

The morphology (color) and mating behavior of male guppies (Poecilia reticulata) in Trinidad reflect a balance between natural selection for cryptic coloration and behavior versus sexual selection for conspicuous coloration and mating behavior (Endler 1992). In the absence of predators, females prefer males with bright coloration and conspicuous displays; thus, behavioral drive by females controls the evolution of male traits in predator-free streams. However, when visually hunting predators (Crenicichla) were experimentally introduced into such streams, they predominantly attacked the most brightly colored and conspicuously displaying males (Endler 1980). Thus, predators selected simultaneously on male color and behavior, and males quickly evolved muted colors and displays (Houde and Endler 1990). The introduction of predators in turn led to a change in female preference against males with bright colors and conspicuous displays (Breden and Stoner 1987; Houde and Endler 1990). Thus, evolutionary changes in morphology (male coloration) and in behavior (male displays) apparently drove a correlated evolutionary change in behavior (female preference). In this example, male morphology and behavior evolved in tandem, and both drove a correlated change in behavior (female preference).

Other examples of behavior and morphology evolving synchronously rather than sequentially are known (Brodie 1992; Wimberger and de Queiroz 1996). For example, garter snakes (Thamnophis ordinoides) are polymorphic both in coloration (striped vs. blotched) and in antipredator behavior (fleeing in a direct line at high speed vs. rapidly reversing direction and freezing). Striped morphs that flee at high speed in a direct line have a higher probability of survival than those that reverse direction; conversely, blotched morphs that reverse direction have a higher probability of survival than do those that flee in a straight line (Brodie 1992). In these snakes, behavior and morphology coevolve- a phenomenon referred to as "correlational selection" (Brodie 1992). In the cases of correlational selection, genes for behavior and morphology become genetically correlated through a synergistic interaction involving prey traits (e.g., morphology and color) and predator sensory systems (Sinervo and Svensson 2002).
Behavior can sometimes constrain or canalize the trajectories of trait evolution along specific lines. For example, the sensory (i.e., behavioral) capacities of particular species bias or constrain the likely direction of evolutionary change in other traits (Burley 1986; Ryan and Rand 1990, 1993; Endler 1992; Ryan 1998); this phenomenon has been called "sensory drive" (Endler 1992) or "receiver bias" (Ryan 1990). For example, females may have "preexisting biases" favoring a particular male coloration that initially enhanced feeding success or predator avoidance (Basolo 1990). Once those biases are established, females will choose males with the preferred colors. Thus, although behavior (female choice) drives the evolution of male morphology, the actual evolutionary trajectory followed by male morphology is simultaneously constrained by female sensory bias. In this case, selection fosters a genetic correlation between female behavior and male morphology, and that genetic correlation fuels a runaway process. Thus, behavior and morphology undergo a synergistic form of correlational selection.

Sensory bias appears important in the evolution of aposematic (warning) coloration and of mimicry complexes as well as in sexual selection (Burley 1986; Ryan and Rand 1990; Endler 1992). Predators learn to avoid distasteful (unprofitable) prey more quickly if it is conspicuous to them (Gittleman et al. 1980; Guilford 1988); this sensory bias of the predator can thus restrict options for effective aposematic coloration. Similarly, the innate bias that some predators have against prey with particular color patterns (Smith 1977) could easily channel the evolutionary trajectory of aposematic coloration or mimetic coloration.

Finally, we note that behavioral inertia is directly relevant to interpreting experiments that use "laboratory natural selection" (Rose et al. 1987). For example, to determine whether organisms have the genetic potential to adapt to climate change, multiple labs have forced organisms to evolve for extended periods at different fixed temperatures (Powell 1974; Huey et al. 1991; Bennett and Lenski 1993; Cavicchi et al. 1995; Partridge et al. 1995). In these studies, the organisms were unable to use thermoregulatory behaviors and thus were forced to be at experimenter-imposed temperatures (Huey et al. 1991). Perhaps not surprisingly, most examined traits (including morphology, life history, physiological characteristics) evolved rapidly. However, had the experimenters used protocols (see Davis et al. 1995) that did not restrict the use of thermoregulatory behaviors, the rates of evolution of other traits probably would have been slower. Thus, laboratory selection experiments generate rapid responses to selection by preventing organisms from using regulatory behaviors that will buffer environmental conditions. Consequently, the results of these experiments may overesti- 
mate how quickly organisms will actually evolve in nature (Huey and Kingsolver 1993).

\section{Concluding—if Complicating—Remarks}

Our goal here is neither to deny nor to challenge Mayr's assertion $(1959,1963)$ that behavior usually drives evolutionary change; an evaluation of that assertion requires a balanced and quantitative review of available evidence (see Plotkin 1988; Wcislo 1989; West-Eberhard 1989; Gittleman et al. 1996). Instead, our main goal is to develop and apply a null model that shows quantitatively that one class of behaviors ("regulatory") sometimes inhibit rather than drive evolutionary change. This is hardly a novel idea (Bogert 1949), but the addition of a null-model approach is.

Our secondary goal is to review a few examples of scenarios that run counter to behavioral drive. For example, some studies show that selection on morphology can drive behavioral change ("morphological drive"), that sensory bias can constrain the direction of morphological change, and that behavior and morphology sometimes evolve concordantly (thus, one does not drive the other). Such concordant evolution should be a general outcome whenever two players (signaler and receiver) interact (as in sexual selection or predator-prey relationships) in a frequencydependent fashion (Sinervo and Svensson 2002).

In closing, we emphasize that the role of behavior in evolution should not be reduced to an "either/or" debate. In fact, we would argue that it is logically inappropriate to ask, Does behavior primarily drive or inhibit evolutionary change in other traits? The example of lizards on an altitudinal gradient is instructive here. We have noted, as Bogert $(1949,1959)$ did, that behavioral shifts effectively buffer natural selection for local physiological adaptation to the thermal environment. In effect, these behavioral shifts promote physiological homeostasis. Yet as Lewontin (1983) cautions, homeostasis in one trait can be achieved only by "nonhomeostasis" in other traits. In the present example, homeostasis of body temperature across an altitudinal gradient is achieved by shifting behaviors and perhaps by changes in morphological traits such as size, shape, and color (Bogert 1949, 1959). For example, highaltitude lizards achieve warm body temperatures by shifting into open habitats and onto horizontal logs or on the ground, whereas low-altitude lizards typically perch on vertical tree trunks off the ground and in shade (Rand 1964; Schoener and Schoener 1971; Huey and Webster 1976; Hertz 1992b). These behavioral shifts, while buffering selection on thermal physiology (fig. 2), may simultaneously drive selection for morphological shifts that facilitate locomotion in different substrates (Bogert 1949, 1959; Moermond 1979; Losos and Sinervo 1991; Sinervo and Losos 1991) or for coloration that promotes crypsis in a different light environment (Endler 1978). In other words, behavior can buffer evolution of one trait while simultaneously driving the evolution of others (Bogert 1949, 1959).

Behavior clearly has multiple, interacting roles in evolution. Thus, an exploration of the roles of behavior in evolution must involve an exploration of the vectors and tensions that link these multidimensional roles. We argue that a null model approach may be an effective means to resolve the relative role of behavior in governing the intensity of selection and thus the rate of evolutionary change.

\section{Acknowledgments}

We thank D. Bauwens, D. Berrigan, F. Breden, A. Clarke, A. Dunham, J. Endler, J. Felsenstein, C. Gans, J. Gittleman, B. Grant, J. Kingsolver, G. Orians, C. R. Peterson, S. Stearns, R. Stevenson, and D. Wake for enlightening discussion on relevant topics. We thank S. Stearns, J. Travis, and an anonymous reviewer for constructive comments on the manuscript. Our research was supported by National Science Foundation grants to R.B.H. (DEB 9981598), P.E.H. (BSR 82-18878), and B.S. (DEB 0108577).

\section{Literature Cited}

Aboitiz, F. 1990. Behavior, body types and the irreversibility of evolution. Acta Biotheoretica 38:91-101.

Adolph, S. C. 1990a. Influence of behavioral thermoregulation on microhabitat use by two Sceloporus lizards. Ecology 71:315-327.

- 1990b. Perch height selection by juvenile Sceloporus lizards: interspecific differences and relationship to habitat use. Journal of Herpetology 24:69-75.

Andersson, M. 1994. Sexual selection. Princeton University Press, Princeton, N.J.

Avery, R. A. 1982. Field studies of body temperatures and thermoregulation. Pages 93-166 in C. Gans and F. H. Pough, eds. Biology of the Reptilia. Vol. 12. Physiology (C). Academic Press, London.

Bakken, G. S. 1992. Measurement and application of operative and standard operative temperatures in ecology. American Zoologist 32:194-216.

Bartholomew, G. A. 1964. The roles of physiology and behaviour in the maintenance of homeostasis in the desert environment. Symposia of the Society for Experimental Biology 18:7-29.

Basolo, A. L. 1990. Female preference predates the evolution of the sword in swordtail fish. Science (Washington, D.C.) 250:808-810.

Bateson, P. 1988. The active role of behaviour in evolution. 
Pages 191-207 in M.-W. Ho and S. W. Fox, eds. Evolutionary processes and metaphors. Wiley, New York.

Bennett, A. F. 1980. The thermal dependence of lizard behaviour. Animal Behaviour 26:455-462.

Bennett, A. F., and R. B. Huey. 1990. Studying the evolution of physiological performance. Pages 251-284 in D. J. Futuyma and J. Antonovics, eds. Oxford surveys in evolutionary biology. Vol. 7. Oxford University Press, Oxford.

Bennett, A. F., and R. E. Lenski. 1993. Evolutionary adaptation to temperature. II. Thermal niches of experimental lines of Escherichia coli. Evolution 47:1-12.

Bennett, A. F., K. M. Dao, and R. E. Lenski. 1990. Rapid evolution in response to high temperature selection. $\mathrm{Na}$ ture 346:79-81.

Boake, C. R. B. 1994. Behavior variation and speciation: flexibility may be a constraint. Pages 259-268 in R. J. Greenspan and C. P. Kyriacou, eds. Flexibility and constraint in behavioral systems. Wiley, New York.

Bogert, C. M. 1949. Thermoregulation in reptiles, a factor in evolution. Evolution 3:195-211.

- 1959. How reptiles regulate their body temperature. Scientific American 200:105-120.

- 1980. Investigations in the field and laboratory of behavioural thermoregulation in an iguanid lizard. Pages 90-102 in T. T. P. Gunawardana, L. Prematilleka, and R. Silva, eds. P. E. P. Deraniyagala commemoration volume. Lake House Investments, Colombo.

Brandon, R. N. 1988. The levels of selection: a hierarchy of interactors. Pages 51-71 in H. C. Plotkin, ed. The role of behavior in evolution. MIT Press, Cambridge, Mass.

. 1990. Adaptation and environment. Princeton University Press, Princeton, N.J.

Brattstrom, B. H. 1965. Body temperature of reptiles. American Midland Naturalist 73:376-422.

Breden, F., and G. Stoner. 1987. Male predation risk determines female preferences in the Trinidad guppy. Nature 329:831-835.

Brodie, E. D., III. 1992. Correlational selection for color pattern and antipredator behavior in the garter snake Thamnophis ordinoides. Evolution 46:1284-1298.

Burley, N. 1986. Comparison of the band color preferences of two species of estrildid finch. Animal Behaviour 34: 1732-1741.

Cavicchi, S., D. Guerra, V. LaTorre, and R. B. Huey. 1995. Chromosomal analysis of heat-shock tolerance in Drosophila melanogaster evolving at different temperatures in the laboratory. Evolution 49:676-684.

Christian, K. A., and C. R. Tracy. 1981. The effect of the thermal environment on the ability of hatchling Galapagos land iguanas to avoid predation during dispersal. Oecologia (Berlin) 49:218-223.
Cowles, R. B., and C. M. Bogert. 1944. A preliminary study of the thermal requirements of desert reptiles. Bulletin of the American Museum of Natural History 83: 261-296.

Coyne, J. A., J. Bundgaard, and T. Prout. 1983. Geographic variation of tolerance to environmental stress in Drosophila pseudoobscura. American Naturalist 122: 474-488.

Darwin, C. D. 1859. The origin of species by means of natural selection, or the preservation of favoured races in the struggle for life. J. Murray, London.

Davis, A. J., L. S. Jenkinson, J. H. Lawton, B. Shorrocks, and S. Wood. 1995. Global warming, population dynamics and community structure in a model insect assemblage. Pages 431-439 in R. Harrington and N. E. Stork, eds. Insects in a changing environment. Harcourt Brace, London.

Elton, C. 1927. Animal ecology. Sidgwick \& Jackson, London.

Endler, J. A. 1978. A predator's view of animal color patterns. Evolutionary Biology 11:319-364.

-1980. Natural selection on color patterns in Poecilia reticulata. Evolution 34:76-91.

- 1992. Signals, signal conditions, and the direction of evolution. American Naturalist 139(suppl.): S125-S153.

Endler, J. A., and T. McLellan. 1988. The process of evolution: towards a newer synthesis. Annual Review of Ecology and Systematics 19:395-421.

Gingerich, P. D. 1983. Rates of evolution: effects of time and temporal scaling. Science (Washington, D.C.) 222: 159-161.

Gittleman, J. L., P. H. Harvey, and P. J. Greenwood. 1980. The evolution of conspicuous coloration: some experiments in bad taste. Animal Behaviour 28:897-899.

Gittleman, J. L., C. G. Anderson, M. Kot, and H.-K. Luh. 1996. Phylogenetic lability and rates of evolution: a comparison of behavioral, morphological and life history traits. Pages 166-205 in E. P. Martins, ed. Phylogenies and the comparative method in animal behavior. Oxford University Press, New York.

Grant, B. W., and A. E. Dunham. 1990. Elevational covariation in environmental constraints and life histories of the desert lizard Sceloporus merriami. Ecology 71: $1765-1776$.

Guilford, T. 1988. The evolution of conspicuous coloration. American Naturalist 131(suppl.):S7-S21.

Haldane, J. B. S. 1949. Suggestions as to the quantitative measurement of rates of evolution. Evolution 3:51-56.

Hardy, A. 1965. The living stream: evolution and man. Harper \& Row, New York.

Harvey, P. E., and M. P. Pagel. 1991. The comparative 
method in evolutionary biology. Oxford University Press, Oxford.

Heath, J. E. 1964. Reptilian thermoregulation: evaluation of field studies. Science (Washington, D.C.) 145: 784-785.

. 1965. Temperature regulation and diurnal activity in horned lizards. University of California Publications in Zoology 64:97-136.

Hedgecock, E. M., and R. L. Russell. 1975. Normal and mutant thermotaxis in the nematode Caenorhabditis elegans. Proceedings of the National Academy of Sciences of the USA 72:4061-4065.

Hertz, P. E. 1981. Adaptation to altitude in two West Indian anoles (Reptilia: Iguanidae): field thermal biology and physiological ecology. Journal of Zoology (London) 195: 25-37.

- 1992a. Evaluating thermal resource partitioning by sympatric Anolis cooki and A. cristatellus: a field test using null hypotheses. Oecologia (Berlin) 90:127-136.

— 1992b. Temperature regulation in Puerto Rican Anolis lizards: a field test using null hypotheses. Ecology 73:1405-1417.

Hertz, P. E., and R. B. Huey. 1981. Compensation for altitudinal changes in the thermal environment by some Anolis lizards on Hispaniola. Ecology 62:515-521.

Hertz, P. E., R. B. Huey, and E. Nevo. 1983. Homage to Santa Anita: thermal sensitivity of sprint speed in agamid lizards. Evolution 37:1075-1084.

Hertz, P. E., R. B. Huey, and R. D. Stevenson. 1993. Evaluating temperature regulation by field-active ectotherms: the fallacy of the inappropriate question. American Naturalist 142:796-818.

Houde, A. E., and J. A. Endler. 1990. Correlated evolution of female mating preferences and male color patterns in the guppy Poecilia reticulata. Science (Washington, D.C.) 248:1405-1408.

Huey, R. B. 1982. Temperature, physiology, and the ecology of reptiles. Pages 25-91 in C. Gans and F. H. Pough, eds. Biology of the Reptilia. Vol. 12. Physiology (C). Academic Press, London.

- 1983. Natural variation in body temperature and physiological performance in a lizard. Pages 484-490 in A. G. J. Rhodin and K. Miyata, eds. Advances in herpetology and evolutionary biology: essays in honor of Ernest E. Williams. Museum of Comparative Zoology, Cambridge, Mass.

Huey, R. B., and A. F. Bennett. 1987. Phylogenetic studies of coadaptation: preferred temperatures versus optimal performance temperatures of lizards. Evolution 41: 1098-1115.

Huey, R. B., and D. Berrigan. 2001. Temperature, demography, and ectotherm fitness. American Naturalist 158: 204-210.
Huey, R. B., and J. G. Kingsolver. 1993. Evolution of resistance to high temperature in ectotherms. American Naturalist 142(suppl.):S21-S46.

Huey, R. B., and R. D. Stevenson. 1979. Integrating thermal physiology and ecology of ectotherms: a discussion of approaches. American Zoologist 19:357-366.

Huey, R. B., and T. P. Webster. 1976. Thermal biology of Anolis lizards in a complex fauna: the cristatellus group on Puerto Rico. Ecology 57:985-994.

Huey, R. B., E. R. Pianka, and J. Hoffmann. 1977. Seasonal variation in thermoregulatory behavior and body temperatures of diurnal Kalahari lizards. Ecology 58: 1066-1075.

Huey, R. B., L. Partridge, and K. Fowler. 1991. Thermal sensitivity of Drosophila melanogaster responds rapidly to laboratory natural selection. Evolution 45:751-756.

Jayne, B. C., and A. F. Bennett. 1990. Selection on locomotor performance capacity in a natural population of garter snakes. Evolution 44:1204-1229.

Jones, J. S., J. A. Coyne, and L. Partridge. 1987. Estimation of the thermal niche of Drosophila melanogaster using a temperature-sensitive mutant. American Naturalist 130:83-90.

Kiester, A. R., G. C. Gorman, and D. C. Arroyo. 1975. Habitat selection behavior of three species of Anolis lizards. Ecology 56:220-225.

Kirkpatrick, M. 1982. Sexual selection and the evolution of female choice. Evolution 36:1-12.

Lamarck, J. B. 1809. Philosophie zoologique. Dentu, Paris. Lande, R. 1981. Models of speciation by sexual selection on polygenic traits. Proceedings of the National Academy of Sciences of the USA 78:3721-3725.

Levinton, J. 1988. Genetics, paleontology, and macroevolution. Cambridge University Press, Cambridge.

Lewontin, R. C. 1983. The organism as the subject and object of evolution. Scientia 118:63-82.

Losos, J. B., and B. Sinervo. 1991. The effects of morphology and perch diameter on sprint performance of Anolis lizards. Journal of Experimental Biology 145: 23-30.

Mayr, E. 1959. The emergence of evolutionary novelties. Pages 349-380 in S. Tax, ed. Evolution after Darwin. University of Chicago Press, Chicago.

- 1963. Animal species and evolution. Harvard University Press, Cambridge, Mass.

- 1974. Behavior programs and evolutionary strategies. American Scientist 62:650-659.

Moermond, T. 1979. The influence of habitat structure on Anolis foraging behavior. Behaviour 70:147-167.

Odling-Smee, F. J. 1988. Niche-constructing phenotypes. Pages 73-131 in H. C. Plotkin, ed. The role of behavior in evolution. MIT Press, Cambridge, Mass.

Partridge, L., B. Barrie, N. H. Barton, K. Fowler, and V. 
French. 1995. Rapid laboratory evolution of adult life history traits in Drosophila melanogaster in response to temperature. Evolution 49:538-544.

Plotkin, H. C. 1988. Behavior and evolution. Pages 1-17 in $\mathrm{H}$. C. Plotkin, ed. The role of behavior in evolution. MIT Press, Cambridge, Mass.

Porter, W. P. 1989. New animal models and experiments for calculating growth potential at different elevations. Physiological Zoology 62:286-313.

Porter, W. P., and C. R. Tracy. 1983. Biophysical analyses of energetics, time-space utilization, and distributional limits. Pages 55-83 in R. B. Huey, E. R. Pianka, and T. W. Schoener, eds. Lizard ecology: studies of a model organism. Harvard University Press, Cambridge, Mass.

Powell, J. R. 1974. Temperature-related genetic divergence in Drosophila body size. Journal of Heredity 65:257-258.

Rand, A. S. 1964. Ecological distribution in anoline lizards of Puerto Rico. Ecology 45:745-752.

Rose, M. R., P. M. Service, and E. W. Hutchinson. 1987. Three approaches to trade-offs in life-history evolution. Pages 91-105 in V. Loeschcke, ed. Genetic constraints on adaptive evolution. Springer, Berlin.

Roughgarden, J., W. P. Porter, and D. Heckel. 1981. Resource partitioning of space and its relationship to body temperatures in Anolis lizard populations. Oecologia (Berlin) 50:256-264.

Ryan, M. J. 1990. Sexual selection, sensory systems, and sensory exploitation. Oxford Surveys in Evolutionary Biology 7:156-195.

- 1998. Sexual selection, receiver biases, and the evolution of sex differences. Science (Washington, D.C.) 281:1999-2003.

Ryan, M. J., and A. S. Rand. 1990. The sensory basis of sexual selection for complex calls in the Túngara frog, Physalaemus pustolusus (sexual selection for sensory exploitation). Evolution 44:305-314.

. 1993. Sexual selection and signal evolution: the ghost of biases past. Philosophical Transactions of the Royal Society of London B, Biological Sciences 340: 187-195.

Sayeed, O., and B. Benzer. 1996. Behavioral genetics of thermosensation. Proceedings of the National Academy of Sciences of the USA 93:6079-6084.

Schmalhausen, I. I. 1949. The factors of evolution. Blakeston, Philadephia.

Schoener, T. W., and A. Schoener. 1971. Structural habitats of West Indian Anolis lizards. II. Puerto Rican uplands. Breviora 35:1-39.

Sinervo, B., and J. B. Losos. 1991. Walking the tight rope: arboreal sprint performance among Sceloporus occidentalis lizard populations. Ecology 72:1225-1233.

Sinervo, B., and E. Svensson. 2002. Correlational selection and the evolution of genomic architecture. Heredity 89: 329-338.

Smith, S. M. 1977. Coral-snake pattern recognition and stimulus generalization by naive great kiskadees (Aves: Tyrannidae). Nature 265:535-536.

Stearns, S. C., and R. F. Hoekstra. 2000. Evolution: an introduction. Oxford University Press, Oxford.

Stevenson, R. D. 1985. The relative importance of behavioral and physiological adjustments controlling body temperature in terrestrial ectotherms. American Naturalist 126:362-386.

Sultan, S. E., and H. G. Spencer. 2002. Metapopulation structure favors plasticity over local adaptation. American Naturalist 160:271-283.

van Berkum, F. H. 1986. Evolutionary patterns of the thermal sensitivity of sprint speed in Anolis lizards. Evolution 40:594-604.

van Damme, R., D. Bauwens, and R. F. Verheyen. 1990. Evolutionary rigidity of thermal physiology: the case of the cool temperate lizard Lacerta vivipara. Oikos 57: 61-67.

Wake, D. B., G. Roth, and M. H. Wake. 1983. On the problem of stasis in organismal evolution. Journal of Theoretical Biology 101:211-224.

Wcislo, W. T. 1989. Behavioral environments and evolutionary change. Annual Review of Ecology and Systematics 20:137-169.

West-Eberhard, M. J. 1989. Phenotypic plasticity and the origins of diversity. Annual Review of Ecology and Systematics 20:249-279.

Willis, C. A., and S. J. Beaupre. 2000. An application of randomization for detecting evidence of thermoregulation in timber rattlesnakes (Crotalus horridus) from northwestern Arkansas. Physiological and Biochemical Zoology 73:325-334.

Wimberger, P. H., and A. de Queiroz. 1996. Comparing behavioral and morphological characters as indicators of phylogeny. Pages 206-233 in E. P. Martins, ed. Phylogenies and the comparative method in animal behavior. Oxford University Press, New York.

Wyles, J. S., J. G. Kunkel, and A. C. Wilson. 1983. Birds, behavior, and anatomical evolution. Proceedings of the National Academy of Sciences of the USA 80: 4394-4397. 